\title{
Effect of a brief patient communication teaching on both attendee and non-attendee family medicine residents
}

\author{
Dilip Nair, MD¹, Adrienne Mays, MD', Mohit Harsh, MD¹
}

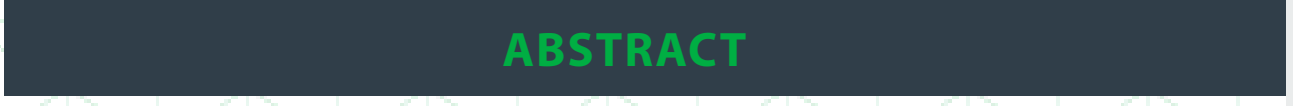

Physician-patient communication skills are important for physicians to acquire. Teaching skills is thought to require attendance by learners but this is difficult in graduate medical education settings. We asked if an educational intervention on physician-patient communication was associated with a "spill-over" effect to non-attendees in the same family medicine residency program.

We surveyed residents regarding communicating instructions to patients before the intervention and one month later, regardless of their attendance. Residents' assessments of their patients' understanding increased significantly post-intervention only if nonattendees were included.

This pilot study suggests a beneficial "spill-over" effect to non-attendee residents that warrants further study.

\section{KEYWORDS}

patient communication, teach back method, residency education

\section{INTRODUCTION}

Communicating effectively with patients is a critical aspect of being a physician. Much research has been done demonstrating that effective physician-patient communication has beneficial effects on health of patients. ${ }^{1}$ As such, communication skills are an important part of graduate medical education. Some have asserted that communication skills can be taught as "verbal procedures" much like other more traditionally instrumental procedures. ${ }^{2}$ Previously published reports on teaching communication skills to residents have contained examples of both single workshops ${ }^{3}$ and multiple briefer sessions. ${ }^{4,5}$ Regardless of the teaching method, resident attendance at the educational intervention is a critical aspect of success. This is particularly important when the intervention is designed to teach a skill. However, due to a variety of factors, resident attendance at teaching conferences is typically so sub-optimal that it has been the subject of much discussion. ${ }^{6,7}$
Author affiliations are listed at the end of this article.

Correspondence to: Hisham Hirzallah, MD Marshall University Joan C. Edwards School of Medicine hirzallah@marshall.edu
If non-attendee residents were able to derive benefit from an educational intervention, that is if a "spill-over effect" existed, then this would suggest effective informal modes of education which might be "harnessed" in more intentional ways to enhance formal educational interventions.

\section{ObJeCTIVE}

Our objective was to study the effect of a patientcentered communication educational intervention on the knowledge, attitudes and practice of family medicine residents in our program. In particular, we planned to study the effect of the intervention on even those residents who did not attend the intervention to determine if they would experience a "spill-over" effect. To our knowledge no other investigation has included this latter component. 


\section{METHODS}

In 2015, we obtained permission from our institution's review board (IRB) to conduct a research project involving the residents of our medical school's family medicine residency program where two of us serve as faculty members. For a patientcentered communication skill on which to focus our intervention, we selected the Teach-Back Method (TBM). This method, in which physicians assess the effectiveness of their instructions by asking the patient for their understanding in their own words, has been shown to be associated with improvement of patient-oriented clinical outcomes. ${ }^{8}$

\section{INTERVENTION}

Our 90-minute intervention, held at our departmental weekly grand rounds meeting, consisted of two sections. First, one of us gave a 20-minute lecture defining the TBM, explaining when and how to use it in the course of patientcentered communication and providing evidence for TBM's efficacy. Next, we engaged the residents around four physician-patient role-play scenarios. Each was designed to address a patient whose characteristics would pose a different challenge in patientcentered communication: low health literacy, impairing comorbidities, complex medical situations and reluctance to access the healthcare system. Resident volunteers took turns playing the role of physician communicating with the patient, played by one faculty member, while the other faculty member guided a group discussion of the interaction.

\section{Measurement}

In the days preceding the intervention, all the program's residents who were on-site at the time of the study, 20 out of a total complement of 24 (83.33\%), were individually invited by our department research coordinators to complete a written questionnaire. The two research coordinators obtained consent and administered the questionnaires confidentially. The survey began by asking for the definition of the term "Teach-Back Method". The participants then received the correct definition and proceeded to complete four core questions designed for responses on a 5-point Likert scale. (Figure 1) The questions covered three areas: the residents' perceptions of the efficacy of the TBM, their perception of the nature of their current patient communication practices, and of the effectiveness of these practices. One month following the intervention, we presented all the residents who had completed the pre-intervention questionnaire with the identical questionnaire, whether or not they had attended the intervention.

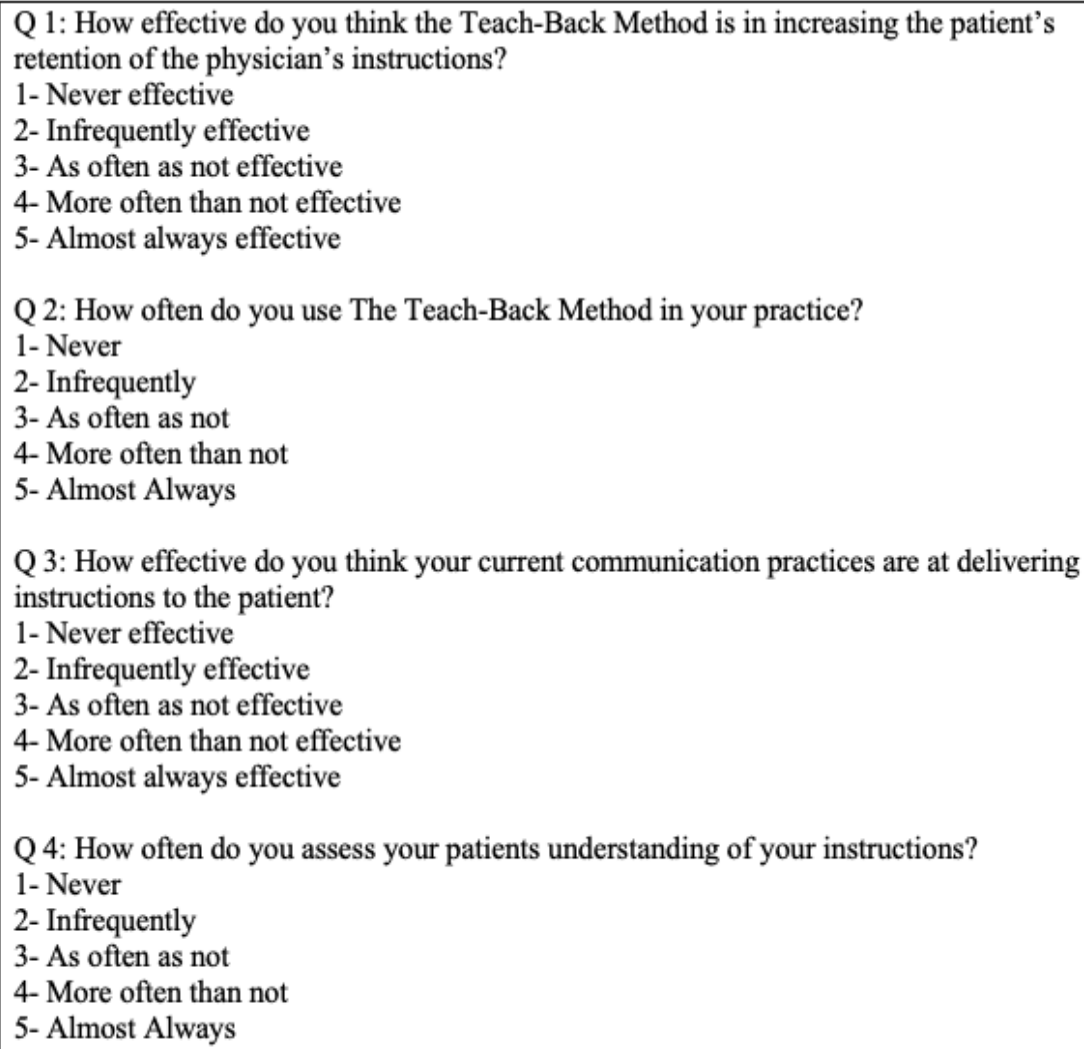

FIGURE 1: Core survey questions 
AnALYSIS

Using Stata 13 (College Station, TX) we made statistical comparisons between the pre- and postintervention study data. In the case of categorical nominal data, we used the Pearson Chi2 test. For continuous data we used the Wilcoxon Signed Rank test instead of the Student's t-test because our sample was not normally distributed.

\section{RESULTS}

All 20 invited residents completed the study questionnaire both before and after the intervention. Of these, 12 residents $(60 \%)$ attended the intervention. A significantly larger proportion of the 12 residents who attended the intervention were able to accurately define the TBM after the intervention than before. Improvement in knowledge was even more notable when the responses of all 20 residents were taken into account; $75 \%$ after intervention compared with 30\% prior to it. (Table 1)
In considering the 12 intervention attendees only, there were no statistically significant changes in their responses to any of the 4 core survey questions. However, when responses from the non-attendees were included, one pre- and post-intervention comparison reached statistical significance; residents reported assessing for the patients' understanding of their communication more frequently. (Table 2 )

\section{DISCUSSION}

The contribution of our pilot project is the potential "spill-over" effect observed. In our study, even the residents who did not attend the intervention perceived themselves as having improved their patient communication one month after the intervention. This "spill-over" effect to nonattendee residents might be due to the survey that all the residents completed beforehand or to communication about the intervention between attendees and non-attendees or some combination of the two. Regardless, if this effect were robust and consistent, it would be important information for postgraduate medical educators.

\begin{tabular}{|l|l|l|l|}
\hline & $\begin{array}{l}\text { Correct Definition } \\
\text { Pre-Intervention (\%) }\end{array}$ & $\begin{array}{l}\text { Correct Definition } \\
\text { Post-Intervention (\%) }\end{array}$ & P value \\
\hline $\begin{array}{l}\text { Attendee } \\
\text { Residents }\end{array}$ & 33.3 & 75 & 0.04 \\
\hline All Residents & 30 & 75 & 0.004 \\
\hline
\end{tabular}

Our pilot study was limited in several ways that will be helpful in guiding future research into this topic. Most importantly, residents' perceptions of improved

TABLE 1. Comparison of correct definition of Teach-Back Method pre- and post-intervention patient communication are susceptible to reporting bias. Therefore, we do not know objectively if our residents did in fact improve their patient communication. A follow-up study might assess changes in residents' communication by surveying patients and by direct observation by faculty. Additionally, the size of the effect was small ( 0.4 points on a 5-point scale). It is well-documented that improvement in physicians' communication is associated

TABLE 2. Comparison of pre- and post-intervention responses to core questions by all participants $(\mathrm{N}=20)$

TBM = Teach-Back Method with patient-centered benefits. 1 However, it is not known how much improvement in physician communication is required to produce clinically significant benefit. Again, a follow-up study that 
assesses patient-oriented outcomes would fill this gap. Further, residents in our study were surveyed one month after the educational intervention. It would be helpful to determine if the "spill-over" effect persists for longer. Finally, the scope of our study was limited. We addressed one aspect of patient-centered communication (patients' comprehension of physicians' instruction) in one relatively small family medicine residency program. Follow-up studies would also need to examine the generalizability of our finding.

\section{CONCLUSION}

Patient-centered communication skills are important for physicians to acquire. Such acquisition is an important element of graduate medical education. The success of educational interventions to teach skills is thought to depend on the residents attending the intervention. High attendance rates are difficult to achieve in residency programs. Our study suggests the possibility that there might be a "spill-over" effect to residents who did not attend the intervention. This effect warrants confirmation and exploration.

\section{AUTHOR AFFILIATIONS}

1. Marshall University Joan C. Edwards School of Medicine, Huntington, West Virginia

\section{REFERENCES}

1. 1. King A, Hoppe RB. “Best Practice” for PatientCentered Communication: A Narrative Review. JGME. 2013;doi:http://dx.doi.org/10.4300/ JGME-D-13-00072.1.

2. Henry SG, Holmboe ES, Frankel RM. Evidence-based competencies for improving communication skills in graduate medical education: A review with suggestions for implementation. Med Teach. 2013;35:395-403.

3. Kripalani S, Weiss BD. Teaching About Health Literacy and Clear Communication. J Gen Intern Med. 2006; 21:888-890.

4. Curtis JR, Back AL, Ford DW et al. Effect of Communication Skills Training for Residents and Nurse Practitioners on Quality of Communication With Patients With Serious IIIness. JAMA. 2013;310(21):2271-2281.

5. Smith L, O'Sullivan P, Lo B, Chen H. An Educational Intervention to Improve Resident Comfort with Communication at the End of Life. J Palliat Care. 2013;16(1):54-59.

6. Fitzgerald JD, Wenger NS. Didactic Teaching Conferences for IM Residents: Who Attends, and Is Attendance Related to Medical Certifying Examination Scores? Acad Med. 2003;78(1):8489.

7. Cosimini MJ, Mackintosh L, Chang TP. Number needed to eat: pizza and resident conference attendance. Med Educ. 2016;50(12):1204-1207.

8. Schillinger D, Piette J, Grumbach K et al. Closing the Loop-Physician Communication With Diabetic Patients Who Have Low Health Literacy. Arch Inter Med. 2003;163:83-90. 